“C 2018 IEEE. Personal use of this material is permitted. Permission from IEEE must be obtained for all other uses, in any current or future media, including reprinting/republishing this material for advertising or promotional purposes, creating new collective works, for resale or redistribution to servers or lists, or reuse of any copyrighted component of this work in other works." 


\title{
State-constrained Control of Single-rod Electrohydraulic Actuator with Parametric Uncertainty and Load Disturbance
}

\author{
Qing $\mathrm{Guo}^{\dagger}$, Member, IEEE, Yi Zhang ${ }^{\dagger}$, Member, IEEE, \\ Branko G. Celler, Fellow, IEEE, Steven W. Su, Member, IEEE
}

\begin{abstract}
The suppression of disturbances under parametric uncertainties is one of the most common control problems in electrohydraulic systems (EHSs), as both disturbances and uncertainties often significantly degrade the tracking performance and bias the load pressure of the electrohydraulic actuator (EHA). This paper presents a state-constrained control of single-rod EHA to restrict the position tracking error to a prescribed accuracy and guarantee the load pressure in the maximal power boundary. Furthermore, a dynamic surface is designed to avoid the explosion of complexity due to the repeatedly calculated differentiations of the virtual control variables in the backstepping iteration. Integrating with disturbance observer and parametric estimation law, this state-constrained controller guarantees the asymptotic convergence of system state error under parametric uncertainties and large load disturbances. The effectiveness of the proposed controller has been demonstrated by a comparative experiment on the motion control of the Two-degree-of-freedom (Two-DOF) robotic arm.
\end{abstract}

Index Terms-Electrohydraulic system, State-constrained control, External load, Parametric uncertainty, Dynamic surface.

\section{INTRODUCTION}

$\mathbf{E}$ LECTROHYDRAULIC servo systems are widely used in mechatronic control engineering as they have a superior load efficiency and large force/torques output. EHSs has been commonly applied in large power systems. However, there are two typical problems associated with EHS control.

One of the fundamental difficulties in electrohydraulic control is the undesirable dynamic behaviours of the designed controller which are due to external load disturbance and the parametric uncertainty in the EHS. The former is caused by the driven force or torque of the mechatronic plant and the latter

Q. Guo and Y. Zhang are with the School of Aeronautics and Astronautics, University of Electronic Science and Technology of China, Chengdu, 611731, China, and Q. Guo is also with State Key Laboratory of Fluid Power \& Mechatronic Systems, Zhejiang University, Hangzhou, China (e-mail: guoqinguestc@uestc.edu.cn, yi.zhang@uestc.edu.cn).

B. Celler is with the School of Electrical Engineering and Telecommunications, University of New South Wales, Sydney, NSW, Australia (e-mail: b.celler@unsw.edu.au).

$\mathrm{S}$. $\mathrm{Su}$ is with Faculty of Engineering and Information Technology, University of Technology Sydney, Sydney, NSW, Australia (e-mail: Steven.Su@uts.edu.au).

This work was supported by National Natural Science Foundation of China (No. 51775089, No. 61305092 and No. 51205045), the Fundamental Research Funds for the Central Universities, China (No. ZYGX2015J118, No. ZYGX2016J160), the Open Foundation of the State Key Laboratory of Fluid Power \& Mechatronic Systems (No. GZKF-201515), and the China Postdoctoral Science Foundation funded project (2017M612950).

$\dagger$ Both of authors equally contribute to this work. is mostly due to unknown viscous damping, load stiffness, variations in control fluid volumes, physical characteristics of valve, bulk modulus and oil temperature variations [1]. Different types of external loads on EHA, are often viewed as zero or unknown constant, even in case of a bounded uncertainty disturbance [2]. Chen [3] proposed a nonlinear disturbance observer integrated with a general nonlinear controller. In practice, the external load may be the largely unknown structural disturbance of EHS, which should be compensated by the constructed controller. Even though the feedback control of EHS may be stable, it is clear that the dynamic performance will decline if the external load increases beyond the maximum load capability of EHA. To address parametric uncertainty, several advanced control methods have also been presented such as robust $H_{\infty}$ controllers [4], output regulation control [5], parametric adaptive controllers [6][7][8], and robust controller with extended state observer [9]. These controllers usually adopted adaptive parametric estimation law (APEL) to estimate the uncertainty parameter. It should be noted that the load disturbance and parametric uncertainty often leads to unexpected chatter, overshooting, and the zero bias of tracking error. Thus, the state-constrained control should be considered in the EHS to guarantee not only the prescribed accuracy of the output tracking error but also the desirable dynamic responses of the specified system state.

Since the output-constrained control has been developed for the nonlinear system by Tee and Ge [10], the state-constrained controller is also one of the most commonly encountered control problems in many mechatronic plants such as the anti-skid braking system [11] and EHS [12]. Subsequently a constrained adaptive robust controller is proposed by $\mathrm{Lu}$ and Yao [13] to achieve the required tracking performances with input saturation and matched uncertainties. As opposed to the general quadratic Lyapunov technique, the state constrained control adopts the barrier Lyapunov function (BLF) [14][15] to restrict the system state to a predefined boundary. Then the well-known backstepping method is often adopted to handle a large class of systems dominated by a called strict feedback form (lower-triangular model) as follows

$$
\left\{\begin{array}{rl}
\dot{x}_{i} & =x_{i+1}+\theta_{i} f_{i}\left(\bar{x}_{i}\right)+d_{i}(x), i=1, \ldots n-1 \\
\dot{x}_{n} & =u+\theta_{n} f_{n}(x)+d_{n}(x), \quad y=x_{1}
\end{array},\right.
$$

where $x=\left[x_{1}, \ldots, x_{n}\right]^{T} \in \mathbb{R}^{n}, y \in \mathbb{R}, u \in \mathbb{R}$ are the state vectors, the output and the control variables of this system, 
$\bar{x}_{i}=\left[x_{1}, \ldots, x_{i}\right]^{T} \in \mathbb{R}^{i}, \theta_{i}$ is the parametric uncertainty, $d_{i}$ is uncertain nonlinearity or external disturbance for $i=1, \ldots n$.

However the common backstepping method exhibits explosion of complexity [16][17] due to the repeatedly calculated differentiations of the virtual control variables. For example, the $i^{\text {th }}$ virtual control is often described as $\alpha_{i}=$ $f\left(y_{d}, x, \dot{\alpha}_{i-1}, \ddot{\alpha}_{i-2}, \ldots, \alpha_{1}^{(i-1)}\right)$ where $y_{d}$ is the demand input and the high-order derivatives $\alpha_{j}^{(i-j)}(i=1, \ldots, n-1, j=$ $1, \ldots, n-1-i)$ are derived in backstepping iteration. These high-order derivatives will amplify noise and uncertainty in the final control $u=f\left(y_{d}, x, \dot{\alpha}_{n-1}\right)$, which easily results into violent control and saturation. To address this problem, the dynamic surface control (DSC) has been proposed as a means to create a stable dynamic surface [18] instead of employing the virtual control derivative. The advantage of DSC is that it eliminates the severe proliferation and singularity of the nonlinear system and guarantees fast state convergence and desirable dynamic performance [11]. The dynamic surface is often designed as a linear filter to transform high order derivatives of virtual control into stable dynamic surfaces.

In this study, based on BLF and dynamic surface techniques, a state-constrained controller is proposed for the single-rod EHA model to achieve the prescribed dynamic tracking performance of the hydraulic cylinder position. Simultaneously, the load pressure is restricted in the maximal power boundary to eliminate the negative impacts of abnormal oil compressibility, hydraulic parametric uncertainty and largely unknown disturbance. The external load from the driven torque of the robotic arm is estimated by a disturbance observer to eliminate the zero bias of the position response. The four uncertainty parameters of EHS are also estimated by a parametric estimation law to reduce the negative effect of the parametric uncertainties. The comparison results with the other two commonly used controllers have verified the effectiveness of the proposed controller in terms of the position tracking performance and the fast response of the hydraulic load pressure.

The remainder of this paper is organized as follows. The plant is described in section II. The state-constrained controller of single-rod EHA is designed in section III including DSC, DO, and APEL. The experimental results demonstrated on the motion of Two-DOF robotic arm are given in section IV. Finally, the conclusion is drawn in section V.

\section{Plant Description}

The EHA is comprised by a servo valve, a single-rod cylinder, a fixed displacement pump, and a relief valve as shown in Fig. 1. The external load on this EHA is a disturbance force which drives the motion control of one robotic arm. The pump outputs the supply pressure $p_{s}$, and the pressure threshold of the relief valve is set as $p_{s}$. As the spool position of the servo valve $x_{v}>0$, the hydraulic oil passes the servo valve and enters the non-rod chamber. The forward channel flow $Q_{a}$ and the cylinder pressure $p_{a}$ are controlled by $x_{v}$. The rod chamber is connected to the return channel and the return pressure is $p_{r}$. On the other hand, the rod chamber is connected to the forward channel where the channel flow $Q_{b}$ and the cylinder pressure $p_{b}$ are controlled by the servo valve

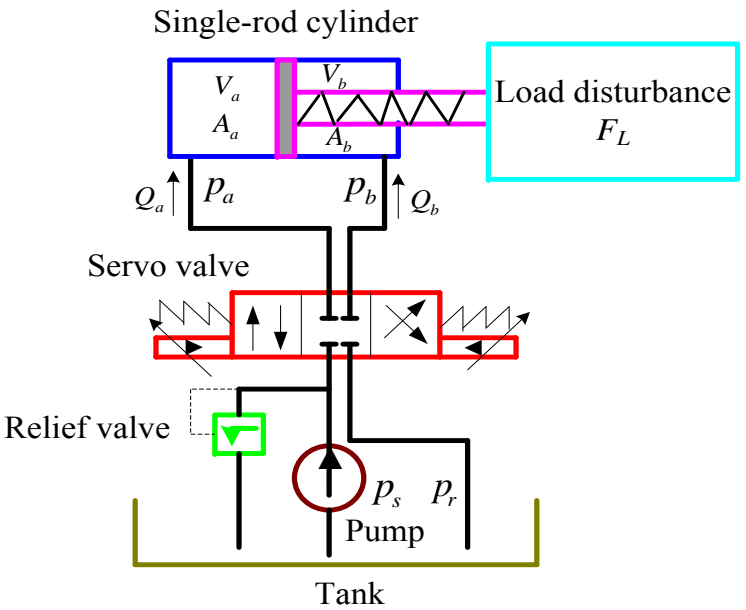

Fig. 1. The single-rod EHA control mechanism

when $x_{v}<0$. The channel flow is cut off as $x_{v}=0$ where the cylinder pressure can be steadily maintained.

Firstly, the load flow equations through the servo valve represent the related model between the channel flow $Q_{i}(i=a, b)$ and the cylinder pressure $p_{i}(i=a, b)$, which are described as follows [1]

$$
\begin{aligned}
& Q_{a}=\left\{\begin{array}{ll}
C_{d} w x_{v} \sqrt{2\left(p_{s}-p_{a}\right) / \rho} & x_{v} \geq 0 \\
C_{d} w x_{v} \sqrt{2\left(p_{a}-p_{r}\right) / \rho} & x_{v}<0 \\
C_{b} w x_{v} \sqrt{2\left(p_{b}-p_{r}\right) / \rho} & x_{v} \geq 0 \\
C_{d} w x_{v} \sqrt{2\left(p_{s}-p_{b}\right) / \rho} & x_{v}<0
\end{array},\right.
\end{aligned},
$$

where $C_{d}$ is the discharge coefficient, $w$ is the area gradient of the servo valve, $\rho$ is the density of the hydraulic oil.

Secondly, according to the flow conservation law, the hydraulic pressure behavior for a compressible fluid volumes, i.e., the flow-pressure continuous model is given by [2][4]

$$
\left\{\begin{array}{l}
A_{a} \dot{y}+C_{t l}\left(p_{a}-p_{b}\right)+\left(V_{0 a}+A_{a} y\right) \dot{p}_{a} / \beta_{e}=Q_{a} \\
A_{b} \dot{y}+C_{t l}\left(p_{a}-p_{b}\right)-\left(V_{0 b}-A_{b} y\right) \dot{p}_{b} / \beta_{e}=Q_{b}
\end{array},\right.
$$

where $y$ is the displacement of piston, $C_{t l}$ is the coefficient of the total leakage of the cylinder, $\beta_{e}$ is the effective bulk modulus, $A_{a}$ and $A_{b}$ are ram areas of the two chambers, $V_{0 a}$ and $V_{0 b}$ are the initial total control volumes of the two cylinder chambers, respectively.

Then from Newton's second law, the mechanical dynamic equation is described as follows [6]

$$
m \ddot{y}=p_{a} A_{a}-p_{b} A_{b}-K y-b \dot{y}-F_{L}(t) .
$$

where $m$ is the load mass, $K$ is the load spring constant, $b$ is the viscous damping coefficient of the hydraulic oil, $F_{L}$ is the external load on the hydraulic actuator.

Without loss of generality, according to the step response and frequency response curve of the Moog servo valve, the dynamics of the servo valve describes the relationship between the spool position and the input control voltage, which is a first order linear model as follows [19]

$$
T_{s v} \dot{x}_{v}+x_{v}=K_{s v} u \text {. }
$$


If the five state variables are defined as $\boldsymbol{X}=$ $\left[x_{1}, x_{2}, x_{3}, x_{4}, x_{5}\right]^{T}=\left[y, \dot{y}, p_{a}, p_{b}, x_{v}\right]^{T}$, and (2) is substituted into (3), then the nonlinear state space model of the single-rod EHA is given by

$$
\left\{\begin{aligned}
\dot{x}_{1}= & x_{2} \\
\dot{x}_{2}= & \left(x_{3} A_{a}-x_{4} A_{b}-K x_{1}-b x_{2}-F_{L}\right) / m \\
\dot{x}_{3}= & h_{1}\left(-A_{a} x_{2}-C_{t l}\left(x_{3}-x_{4}\right)\right) \\
& +h_{1} C_{d} w x_{5} \sqrt{2 / \rho}\left(s_{1} \sqrt{p_{s}-x_{3}}+s_{2} \sqrt{x_{3}-p_{r}}\right) \\
\dot{x}_{4}= & h_{2}\left(A_{b} x_{2}+C_{t l}\left(x_{3}-x_{4}\right)\right) \\
& -h_{2} C_{d} w x_{5} \sqrt{2 / \rho}\left(s_{1} \sqrt{x_{4}-p_{r}}-s_{2} \sqrt{p_{s}-x_{4}}\right) \\
\dot{x}_{5}= & -x_{5} / T_{s v}+K_{s v} u / T_{s v}
\end{aligned}\right.
$$

where $h_{1}=\beta_{e} /\left(V_{0 a}+A_{a} x_{1}\right), h_{2}=\beta_{e} /\left(V_{0 b}-A_{b} x_{1}\right), s_{1}=$ $1+\operatorname{sgn}\left(x_{5}\right) / 2, s_{2}=1-\operatorname{sgn}\left(x_{5}\right) / 2$.

Remark 1: [8] Without loss of generality, the above hydraulic parameters $b, C_{t l}, C_{d}, w, \rho$ are unknown constants.

Remark 2: [12] The external load $F_{L}$ is a structural disturbance of EHS. Although the dynamic value of $F_{L}$ depends on the variables $y, \dot{y}, \ddot{y}$, it is bounded by its upper boundary $\Delta_{F L}$, i.e., $\left\|F_{L}(t)\right\|<\Delta_{F L}$.

\section{StATE-CONSTRAINED CONTROL OF EHA}

Since the EHA model (6) includes parametric uncertainty and load disturbance, the state-constrained controller is designed by BLF with parametric and disturbance estimations.

\section{A. Strict Feedback Model Construction}

The single-rod model of EHA (6) has one internal dynamic, which means that it is not a strict feedback form as shown in (1). Thus, two new states $\bar{x}_{3}=x_{3}-v x_{4}\left(v=A_{b} / A_{a}<1\right)$, $\bar{x}_{4}=x_{5}$ are defined to guarantee the strict feedback form as follows

$$
\left\{\begin{array}{rl}
\dot{x}_{1}= & x_{2} \\
\dot{x}_{2}= & f_{21}\left(x_{1}\right)+\theta_{1} f_{22}\left(x_{2}\right)+g_{2} \bar{x}_{3}+d(t) \\
\dot{\bar{x}}_{3}= & \theta_{2} f_{31}\left(x_{1}, x_{2}\right)+\theta_{3} f_{32}\left(x_{1}, x_{3}, x_{4}\right) \\
& +\theta_{4} g_{3}\left(x_{1}, x_{3}, x_{4}\right) \bar{x}_{4} \\
\dot{\bar{x}}_{4}= & f_{4}\left(\bar{x}_{4}\right)+g_{4} u
\end{array},\right.
$$

where $\theta_{1}=b, \theta_{2}=\beta_{e}, \theta_{3}=\beta_{e} C_{t l}, \theta_{4}=$ $\beta_{e} C_{d} w \sqrt{2 / \rho}$ are four uncertainty parameters, and $f_{21}\left(x_{1}\right)=$ $-K x_{1} / m, f_{22}=-x_{2} / m, g_{2}=A_{a} / m, d(t)=$ $-F_{L}(t) / m, f_{31}=-\left(h_{1} A_{a}+h_{2} A_{b} v\right) x_{2} / \beta_{e}, f_{32}=-\left(x_{3}-\right.$ $\left.x_{4}\right)\left(h_{1}+h_{2}\right) / \beta_{e}, g_{3}=\bar{s}_{1}\left(h_{1} \sqrt{p_{s}-x_{3}}+v h_{2} \sqrt{x_{4}-p_{r}}\right) / \beta_{e}+$ $\bar{s}_{2}\left(h_{1} \sqrt{x_{3}-p_{r}}+v h_{2} \sqrt{p_{s}-x_{4}}\right) / \beta_{e}, f_{4}=-\bar{x}_{4} / T_{s v}, g_{4}=$ $K_{s v} / T_{s v}, \bar{s}_{1}=\left(1+\tanh \left(k \bar{x}_{4}\right)\right) / 2, \bar{s}_{2}=\left(1-\tanh \left(k \bar{x}_{4}\right)\right) / 2$.

Remark 3: As most functions appearing in the backstepping design are required to be smooth, the $\operatorname{sign}$ function $\operatorname{sgn}(x)$ in (7) is replaced by the hyperbolic tangent function $\tanh (k x)$, where the constant $k>>0$.

Remark 4: Due to abnormal oil compressibility, hydraulic parametric uncertainty and load disturbance, the load pressure $\bar{x}_{3}$ often surpasses its boundary in practice, which declines the dynamic behavior of EHS. Thus, $\bar{x}_{3}$ is bounded by $p_{r}-v p_{s}<$ $\bar{x}_{3}<p_{s}-v p_{r}$ if the two cylinder chamber pressures are $x_{3}$ and $x_{4}$ are bounded by $p_{r}<x_{3}, x_{4}<p_{s}$.
Remark 5: The internal dynamics of EHA (6) can be defined as $\bar{x}_{3}^{\prime}=x_{4}-x_{3} / v$, which is bounded by $p_{r}-p_{s} / v<\bar{x}_{3}^{\prime}<$ $p_{s}-p_{r} / v$. Thus the two dynamics of $\bar{x}_{3}, \bar{x}_{3}^{\prime}$ are equivalent to the state dynamics of $x_{3}, x_{4}$.

\section{B. Disturbance Observer}

The load disturbance $d(t)$ is estimated by a disturbance observer as follow

$$
\hat{d}=K_{d}\left(x_{2}-\xi\right)
$$

where $\hat{d}, \xi$ are the estimations of $d$ and $x_{2}, K_{d}$ is the observer gain.

The dynamics of $\xi$ in (8) is taken as

$$
\dot{\xi}=f_{21}\left(x_{1}\right)+\hat{\theta}_{1} f_{22}\left(x_{2}\right)+g_{2} \bar{x}_{3}+\hat{d},
$$

where $\hat{\theta}_{1}$ is the estimation of $\theta_{1}$.

According to (7), (8) and (9), if the observer error and the parametric estimation error are defined as $\tilde{d}=d-\hat{d}, \tilde{\theta}_{1}=$ $\theta_{1}-\hat{\theta}_{1}$, then the dynamics of $\tilde{d}$ are given by

$$
\dot{\tilde{d}}=-K_{d} \tilde{d}+\dot{d}+K_{d} x_{2} \tilde{\theta}_{1} / m \text {. }
$$

Hence, the observer error $\tilde{d}(t)$ is affected by $\dot{d}$ and $\tilde{\theta}_{1}$. The asymptotic convergence of $\tilde{\theta}_{1}$ can be guaranteed in the controller design subsequently, i.e., $t \rightarrow \infty, \tilde{\theta}_{1} \rightarrow 0$. The convergence of $\tilde{d}(t)$ is discussed in the following two conditions.

1) Due to no prior information about $\dot{d}(t)$, suppose that $\dot{d}(t) \rightarrow 0, t \rightarrow \infty$. From (10), the observer gain $K_{d}>0$ guarantees $\tilde{d}(t) \rightarrow 0, t \rightarrow \infty$.

2) If $\dot{d}(t) \neq 0, \forall t>0$, it is reasonable to assume that $\dot{d}(t)$ is bounded, then $\tilde{d}(t)$ is stable and bounded by $|\tilde{d}| \leq|\dot{d}|_{\max } / K_{d}$. Thus, $\tilde{d}(t)$ can be arbitrarily reduced by increasing the observer gain $K_{d}$.

\section{State-constrained controller}

In (6), two cylinder pressures $p_{a}$ and $p_{b}$ probably surpass the system supply pressure $p_{s}$ and the return pressure $p_{r}$, which will reduce the service life of the EHA in the long term. In practice, the load pressure of cylinder $\bar{x}_{3}$ needs to maintain a certain margin of the power boundary mentioned in Remark 4. Meanwhile, the position tracking accuracy should be the other state-constrained condition in the control design.

The system state errors $z_{i}(i=1, \ldots, 4)$ are defined as

$$
\left\{\begin{array}{rl}
z_{1} & =x_{1}-y_{d} \\
z_{2} & =x_{2}-\alpha_{1} \\
z_{i} & =\bar{x}_{i}-\alpha_{i-1}, i=3,4
\end{array},\right.
$$

where $\alpha_{i}(i=1,2,3)$ are the virtual control variables in the backstepping iteration.

Remark 6: The virtual control $\alpha_{2}$ is considered as $p_{r}<$ $\alpha_{2}<p_{s}$, which converts the constrained state $\bar{x}_{3}$ into the error $z_{3}$. According to Remark 4 , the state-constrained controller adopts the symmetric and asymmetric barrier Lyapunov function (BLF, ABLF) [10] to consider two errors such that

$$
\left\{\begin{array}{c}
-k_{c 1}<z_{1}<k_{c 1} \\
-k_{a 1}<z_{3}<k_{b 1}
\end{array},\right.
$$


where $k_{c 1}$ is the prescribed error boundary of the position tracking error, $k_{a 1}=(v+1) p_{s}-p_{r}$ and $k_{b 1}=p_{s}-(v+1) p_{r}$ are the left and right pressure boundaries of the state error $z_{3}$, respectively.

Assumption 1: [11] For any $k_{c 1}>0$, the positive constants $Y_{l 0}, Y_{h 0}, Y_{1}, Y_{2}, \delta_{y_{d}}$ exist such that $y_{d}(t)$ and its time derivatives satisfy $Y_{l 0} \leq y_{d}(t) \leq Y_{h 0}$ and $\left|\dot{y}_{d}(t)\right| \leq Y_{1},\left|\ddot{y}_{d}(t)\right| \leq Y_{2}$, $\forall t \geq 0$, implying that these are continuous and available in a compact set $\Omega_{y_{d}}:=\left\{y_{d} \in \mathbb{R}: y_{d}^{2}+\dot{y}_{d}^{2}+\ddot{y}_{d}^{2} \leq \delta_{y_{d}}\right\} \subset \mathbb{R}$.

Assumption 2: [10] The functions $g_{i}(i=2,3,4)$ are positive, and a class of positive constants exists being $g_{i \text { min }}$, $g_{i \max }$ such that $0<g_{i \min } \leq g_{i} \leq g_{i \max }$ for $|x|<k_{c 1}$.

Since the demand position $y_{d}$ is continuous and differentiable, its first and second order derivatives $\dot{y}_{d}, \ddot{y}_{d}$ are reasonably assumed to be bounded. In practice, the hydraulic parameters and state variables in EHA model (7) have definite physical meaning, which indicates the functions $g_{i}(i=2,3,4)$ are bounded with known directions. Thus, Assumptions 1-2 are often satisfied.

Lemma 1: [20] For any positive constants $k_{a 1}, k_{b 1}$ and $k_{c 1}$, let $\Omega_{z}:=\left\{\left[z_{1}, z_{3}\right] \subset \mathbb{R}^{2}:\left|z_{1}\right|<k_{c 1},-k_{a 1}<z_{3}<k_{b 1}\right\}$ and $\Omega_{N}:=\mathbb{R}^{l} \times \Omega_{z} \subset \mathbb{R}^{l+2}$ be open sets. If a generalized error $e$ is defined as $e=\left[z_{2}, z_{4}, \tilde{d}, \tilde{\theta}_{1}, \tilde{\theta}_{2}, \tilde{\theta}_{3}, \tilde{\theta}_{4}\right]$, consider this system

$$
\dot{\zeta}=\psi(t, \zeta)
$$

where $\zeta=\left[e, z_{1}, z_{3}\right]^{T} \subset \Omega_{N}$, and $\psi: \mathbb{R}^{+} \times \Omega_{N} \rightarrow \mathbb{R}^{9}$ is piece wise continuous in $t$ and locally Lipschitz in $\Omega_{z}$, uniformly in $t$, on $\mathbb{R}^{+} \times \Omega_{N}$. If two positive definite functions $V_{1}: \mathbb{R}^{7} \rightarrow \mathbb{R}^{+}, V_{2}: \mathbb{R}^{2} \rightarrow \mathbb{R}^{+}$exists in their respective domains such that

$$
\begin{aligned}
& \gamma_{1}(\|e\|) \leq V_{1}(e) \leq \gamma_{2}(\|e\|), \\
& V_{2}\left(z_{1}, z_{3}\right) \rightarrow \infty,\left\{z_{1} \rightarrow \pm k_{c 1}\right\} \cup\left\{z_{3} \rightarrow-k_{a 1}, z_{3} \rightarrow k_{b 1}\right\}
\end{aligned}
$$

where $\gamma_{1}$ and $\gamma_{2}$ are class $K_{\infty}$ functions. Suppose $V(\zeta)=$ $V_{1}(e)+V_{2}\left(z_{1}, z_{3}\right)$, and $\left[z_{1}(0), z_{3}(0)\right]^{T}$ belongs to the set $\Omega_{z} \in$ $\left(-k_{c 1}, k_{c 1}\right) \cup\left(-k_{a 1}, k_{b 1}\right)$. If the following condition

$$
\dot{V}=\frac{\partial V}{\partial \zeta} \psi \leq-c V+\delta
$$

holds in the set $\zeta \in \Omega_{N}$ where $c$ and $\delta$ are positive constants, then $z_{1}(t)$ and $z_{3}(t)$ remain in the open set $\Omega_{z} \in\left(-k_{c 1}, k_{c 1}\right) \cup$ $\left(-k_{a 1}, k_{b 1}\right), \forall t \in[0,+\infty)$.

The proof of Lemma 1 is realized by Tee et al. [10] by referring to Theorem 54 proposed in Sontag [21]. In this Lemma, the system state error $\zeta$ is divided into two parts, i.e., $\left[z_{1}, z_{3}\right]^{T}$ and $e$, where $z_{1}$ and $z_{3}$ are two constrained states, $e$ are seven free states. To address $z_{1}$ and $z_{3}$, the afterward mentioned BLF and ABLF (the logarithm elements in (18)) are adopted to restrict them in respectively prescribed error boundaries as shown in (12). Simultaneously, the quadratic Lyapunov functions are used to handle the free states $e$ in control design.

Lemma 2: [20] For all $z_{1} \subset \mathbb{R}, k_{c 1}>0$, if $\left|z_{1}\right|<k_{c 1}$, the following inequality holds

$$
\ln \frac{k_{c 1}^{2}}{k_{c 1}^{2}-z_{1}^{2}} \leq \frac{z_{1}^{2}}{k_{c 1}^{2}-z_{1}^{2}} .
$$

The inequality in Lemma 2 represents the potential relation between the BLF and QLF, which can be used in Theorem 1 and deriving the system stability condition (22) from (19), (20), (21) and the designed controller $u$ in (17) later.

In this study, the dynamic surface is designed to avoid the explosion of the virtual control derivative $\dot{\alpha}_{i}$ in the backstepping iteration. Here the dynamic surfaces of $z_{i+1}(i=1,2,3)$ are given by a first-order filter form as follows

$$
\tau_{i} \dot{\alpha}_{i}+\alpha_{i}=\beta_{i}, \quad \alpha_{i}(0)=\beta_{i}(0)
$$

where $\beta_{i}(i=1,2,3)$ are the stabilizing functions to be designed, $\tau_{i}(i=1,2,3)$ are the time constants of the dynamic surfaces.

Therefore, the output error of the dynamic surface $S_{i}=\alpha_{i}-$ $\beta_{i}$ and the derivative $\dot{\alpha}_{i}=-S_{i} / \tau_{i}$ are obtained for $i=1,2,3$.

In (7), there exist four uncertainty parameters $\theta_{i}(i=$ $1, \ldots, 4)$. By referring to the reference [2] [22], a parametric estimation law is designed as follows

$$
\left\{\begin{array}{l}
\dot{\hat{\theta}}_{1}=\operatorname{Proj}_{\hat{\theta}_{1}}\left(z_{2} f_{22} / k_{\theta 1}\right) \\
\dot{\hat{\theta}}_{2}=\operatorname{Proj}_{\hat{\theta}_{2}}\left(\mu f_{31} z_{3} / k_{\theta 2}\right) \\
\dot{\hat{\theta}}_{3}=\operatorname{Proj}_{\hat{\theta}_{3}}\left(\mu f_{32} z_{3} / k_{\theta 3}\right) \\
\dot{\hat{\theta}}_{4}=\operatorname{Proj}_{\hat{\theta}_{4}}\left(\mu g_{3} z_{3} \bar{x}_{4} / k_{\theta 4}\right)
\end{array},\right.
$$

where $\operatorname{Proj}_{\hat{\theta}_{i}}\left(\bullet_{i}\right)$ is a projection mapping defined as follow

$$
\operatorname{Proj}_{\hat{\theta}_{i}}\left(\bullet_{i}\right)=\left\{\begin{array}{ll}
0, & \text { if } \hat{\theta}_{i}=\theta_{i \max }, \text { and } \bullet_{i}>0 \\
0, & \text { if } \hat{\theta}_{i}=\theta_{i \text { min }}, \text { and } \bullet_{i}<0 \\
\bullet_{i}, & \text { otherwise }
\end{array},\right.
$$

and $\mu=\left(1-q\left(z_{3}\right) /\left(k_{a 1}^{2}-z_{3}^{2}\right)+q\left(z_{3}\right) /\left(k_{b 1}^{2}-z_{3}^{2}\right), q\left(z_{3}\right)=1\right.$ if $0<z_{3}<k_{b 1}$, otherwise $q\left(z_{3}\right)=0$.

By using the projection mapping (16), the parametric estimation $\hat{\theta}_{i}$ can be bounded by $\theta_{i \text { min }} \leq \hat{\theta}_{i} \leq \theta_{i \max }$ and $\tilde{\theta}_{i}\left(\bullet_{i}-\hat{\theta}_{i}\right) \leq 0$ for $i=1, \ldots, 4$, where the respective parametric estimation error is $\tilde{\theta}_{i}=\theta_{i}-\hat{\theta}_{i}$.

Based on the calculations of (11), (14), (15), the stateconstrained controller $u$ are designed shown in (17). In fact, this controller is an iterative expression, which can be calculated by using Fig. 2.

$$
\left\{\begin{aligned}
\beta_{1}= & -\left(k_{c_{1}}^{2}-z_{1}^{2}\right) k_{1} z_{1}+\dot{y}_{d}-\frac{\sigma_{1}\left(k_{c_{1}}^{2}-z_{1}^{2}\right)}{2 z_{1}} \\
\beta_{2}= & -\frac{1}{g_{2}}\left(k_{2} z_{2}+\frac{z_{1}}{k_{c_{1}}^{2}-z_{1}^{2}}+f_{21}+\hat{\theta}_{1} f_{22}+\hat{d}\right. \\
& \left.+S_{1} / \tau_{1}-\frac{\sigma_{2}+\sigma_{3}}{2 z_{2}}-\frac{K_{d}\left|f_{22}\right| \sigma_{4}}{2 z_{2}}\right) \\
\beta_{3}= & -\frac{1}{\hat{\theta}_{4} g_{3}}\left(\phi g_{2} z_{2}+\hat{\theta}_{2} f_{31}+\hat{\theta}_{3} f_{32}\right. \\
& \left.+S_{2} / \tau_{2}+k_{3} \phi z_{3}-\frac{\phi \sigma_{5}}{2 z_{3}}\right) \\
S_{i}= & \beta_{i}-\alpha_{i}, \quad i=1,2,3 \\
u= & -\frac{1}{g_{4}}\left(k_{4} z_{4}+\mu \hat{\theta}_{4} g_{3} z_{3}+f_{4}\left(\bar{x}_{4}\right)+\frac{S_{3}}{\tau_{3}}\right)
\end{aligned}\right.
$$

where $\beta_{i}(i=1,2,3)$ are the stabilizing functions of the dynamic surfaces (14), $\phi=1 / \mu, k_{i}(i=1, \ldots, 4)$ are control gains, and $\sigma_{i}(i=1, \ldots, 5)$ are positive constants. 


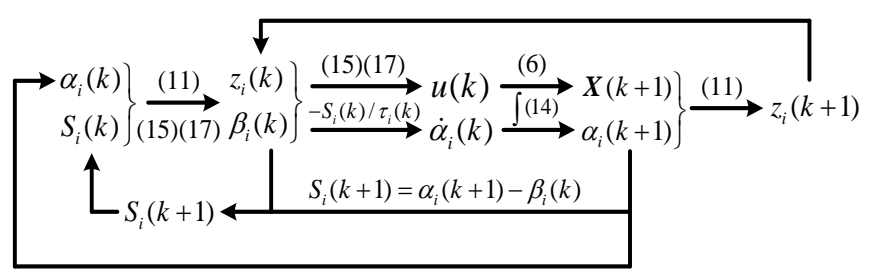

Fig. 2. The iterative calculation of the state-constrained controller

Theorem 1: Considering the dynamic surfaces (14) and the stabilizing functions (17) for the strict feedback model (7) under Assumption 1 and 2, together with the load disturbance observer (8), (9), and the adaptive parametric estimation law (15), if the initial compact set of the partial state errors $z_{1}$ and $z_{3}$ is $\Omega_{z}(0) \subset \Phi_{z}^{0}:=\left\{\Omega_{z}(0):\left|z_{1}\right| \leq k_{c 1},-k_{a 1} \leq\right.$ $\left.z_{3} \leq k_{b 1}\right\}$, then all the generalized errors $Z_{g}(t)$ including $z_{i}$, $\tilde{\theta}_{i}(i=1, \ldots, 4), S_{j}(j=1,2,3)$ and $\tilde{d}$ locally asymptotically converge to zero, i.e., $Z_{g}(t) \rightarrow 0, t \rightarrow \infty$.

Proof. The candidate barrier Lyapunov function of (7) are given by

$$
\begin{aligned}
V= & \frac{1}{2} \ln \frac{k_{c_{1}}^{2}}{k_{c_{1}}^{2}-z_{1}^{2}}+\frac{1}{2} z_{2}^{2}+\frac{1}{2}\left(1-q\left(z_{3}\right)\right) \ln \frac{k_{a 1}^{2}}{k_{a 1}^{2}-z_{3}^{2}} \\
& +\frac{1}{2} q\left(z_{3}\right) \ln \frac{k_{b 1}^{2}}{k_{b 1}^{2}-z_{3}^{2}}+\frac{1}{2} z_{4}^{2} \\
& +\frac{1}{2} \tilde{d}^{2}+\frac{1}{2} \sum_{i=1}^{3} S_{i}^{2}+\frac{1}{2} \sum_{i=1}^{4} k_{\theta_{i}} \tilde{\theta}_{i}^{2}
\end{aligned}
$$

where $k_{\theta i}(i=1, \ldots, 4)$ are the APEL gains.

Since the state $x_{1}$ is a physical variable of the cylinder position, and $\dot{y}_{d}, \ddot{y}_{d}$ are bounded from Assumption $1, \dot{\beta}_{1}$ is also bounded by $\left|\dot{\beta}_{1}\right|_{\max }$. Furthermore, from Assumption 2 and (14), $\beta_{2}$ and $\beta_{3}$ are also bounded. According to Assumption 1 and the physical variables $x_{2}, \bar{x}_{3}, \bar{x}_{4}, \dot{\beta}_{i}$ is also bounded by $\left|\dot{\beta}_{i}\right|_{\max }$ for $i=2,3$.

In terms of the parametric estimation error $\tilde{\theta}_{i}=\theta_{i}-\hat{\theta}_{i}$, since $\hat{\theta}_{i}$ is bounded by $\theta_{i \min } \leq \hat{\theta}_{i} \leq \theta_{i \max }$, then $\left|\tilde{\theta}_{i}\right| \leq$ $\theta_{i \max }-\theta_{i \min }=\Delta \theta_{i \max }$ for $i=1, \ldots, 4$.

Then the following inequalities are obtained via Young's inequality

$$
\begin{aligned}
& \left|z_{i} S_{i}\right| \leq \frac{z_{i}^{2}+S_{i}^{2}}{2}, i=1,2,3, \quad\left|z_{1} \tilde{d}\right| \leq \frac{\tilde{d}^{2}+z_{1}^{2}}{2} \\
& \left|S_{i} \dot{\beta}_{i}\right| \leq \frac{S_{i}^{2}\left|\dot{\beta}_{i}\right|_{\max }^{2}}{2 \sigma_{j}}+\frac{\sigma_{j}}{2}, i=1,2,3, \quad j=2 i-1, \\
& |\tilde{d} \dot{d}| \leq \frac{\tilde{d}^{2}|\dot{d}|_{\max }^{2}}{2 \sigma_{2}}+\frac{\sigma_{2}}{2}, \quad\left|\tilde{d} \tilde{\theta}_{1}\right| \leq \frac{\Delta \tilde{\theta}_{1 \max }^{2} \tilde{d}^{2}}{2 \sigma_{4}}+\frac{\sigma_{4}}{2}
\end{aligned}
$$

From the projection mapping definition in (16), the following inequalities hold

$$
\left\{\begin{array}{l}
\left(z_{2} f_{22}-k_{\theta_{1}} \dot{\hat{\theta}}_{1}\right) \tilde{\theta}_{1} \leq 0 \\
\left(\mu z_{3} f_{31}-k_{\theta_{2}} \dot{\hat{\theta}}_{2}\right) \tilde{\theta}_{2} \leq 0 \\
\left(\mu z_{3} f_{32}-k_{\theta_{3}} \dot{\hat{\theta}}_{3}\right) \tilde{\theta}_{3} \leq 0 \\
\left(\mu g_{3} z_{3} \bar{x}_{4}-k_{\theta_{4}} \dot{\hat{\theta}}_{4}\right) \tilde{\theta}_{4} \leq 0
\end{array}\right.
$$

From Lemma 2, if the following seven positive constants $\Gamma_{i}, \Omega_{i}(i=1,2,3)$ and $\bar{K}_{d}$ are taken as

$$
\begin{aligned}
\Gamma_{1} & =k_{1}\left(k_{c_{1}}^{2}-z_{1}^{2}\right)-\frac{1}{2}>0 \\
\Omega_{1} & =\frac{1}{\tau_{1}}-\frac{1}{2\left(k_{c_{1}}^{2}-z_{1}^{2}\right)}-\frac{\left|\dot{\beta}_{1}\right|_{\text {max }}^{2}}{2 \sigma_{1}}>0 \\
\Gamma_{2} & =k_{2}-\frac{1}{2}-\frac{\left|g_{2}\right|_{\text {max }}^{2}}{2}>0 \\
\Omega_{2} & =\frac{1}{\tau_{2}}-\frac{1}{2 \sigma_{3}}\left|\dot{\beta}_{2}\right|_{\max }^{2}-\frac{\left|g_{2}\right|_{\text {max }}^{2}}{2}>0 \\
\Gamma_{3} & =\left(k_{3}-\mu \theta_{4 \max }\left|g_{3}\right|_{\max } / 2\right) \phi>0 \\
\Omega_{3} & =\frac{1}{\tau_{3}}-\frac{\left|\dot{\beta}_{3}\right|_{\text {max }}^{2}}{2 \sigma_{5}}-\frac{\mu \theta_{4 \max }\left|g_{3}\right|_{\text {max }}}{2}>0 \\
\bar{K}_{d} & =K_{d}-\frac{K_{d}\left|f_{22}\right|_{\max } \Delta \theta_{1 \max }^{2}}{2 \sigma_{4}}-\frac{1}{2}-\frac{1}{2 \sigma_{2}}|\dot{d}|_{\max }^{2}>0
\end{aligned}
$$

Substituting (13), (19), (20), (21), the stabilizing functions $\beta_{i}(i=1,2,3)$ and the control $u$ in (17) into the derivative of the Lyapunov function $V$ in (18), we can see that

$$
\begin{aligned}
\dot{V} \leq & -\Gamma_{1} \ln \frac{k_{c_{1}}^{2}}{k_{c_{1}}^{2}-z_{1}^{2}}-\Gamma_{2} z_{2}^{2}-\bar{K}_{d} \tilde{d}^{2} \\
& -\Gamma_{3}\left(\left(1-q\left(z_{3}\right)\right) \ln \frac{k_{a 1}^{2}}{k_{a 1}^{2}-z_{3}^{2}}+q\left(z_{3}\right) \ln \frac{k_{b 1}^{2}}{k_{b 1}^{2}-z_{3}^{2}}\right) \\
& -\sum_{i=1}^{3} \Omega_{i} S_{i}^{2}-k_{4} z_{4}^{2}<0 .
\end{aligned}
$$

Hence, if the initial system state errors $z_{1}(0)$ and $z_{3}(0)$ belong to the compact set $\Phi_{z}^{0}:=\left\{\Omega_{z}(0):\left|z_{1}\right| \leq k_{c 1},-k_{a 1} \leq\right.$ $\left.z_{3} \leq k_{b 1}\right\}$, then $\dot{V}_{4}(t)<0, \forall t>0$, which indicates that the generalized errors $Z_{g}(t) \rightarrow 0, t \rightarrow \infty$.

From (8) and (15), the convergent rates of the parametric estimation and the disturbance observer can be improved by appropriately regulating the gains $k_{\theta i}(i=1, \ldots, 4)$ and $K_{d}$. They are designed to guarantee that the convergent rates of $\hat{\theta}_{i}(i=1, \ldots, 4)$ and $\hat{d}$ are faster than that of the system state error $z_{i}(i=1, \ldots, 4)$. Then $z_{i}$ can be rapidly reduced by increasing the control gains $k_{i}$ from (17) for $i=1, \ldots, 4$.

Fig. 3 shows the block diagram of the proposed stateconstrained control scheme. The disturbance observer (8) and (9) estimates the unknown external load $F_{L}$. The uncertainty parameters $\theta_{i}(i=1, \ldots, 4)$ are estimated by the parametric estimation law (15). The designed dynamic surface (14) is used to avoid the explosion of complexity. According to the stateconstrained conditions (12), the controller $u$ (17) is constructed based on the BLF to guarantee the dynamic performance of the single-rod EHA (6).

\section{EXPERIMENT}

To verify the effectiveness of the proposed controller (17), the experimental bench of the Two-DOF robotic arm is set up as shown in Fig. 4. The two electrohydraulic actuators include two servo valves (Moog D633-R02K01M0NSM2), 


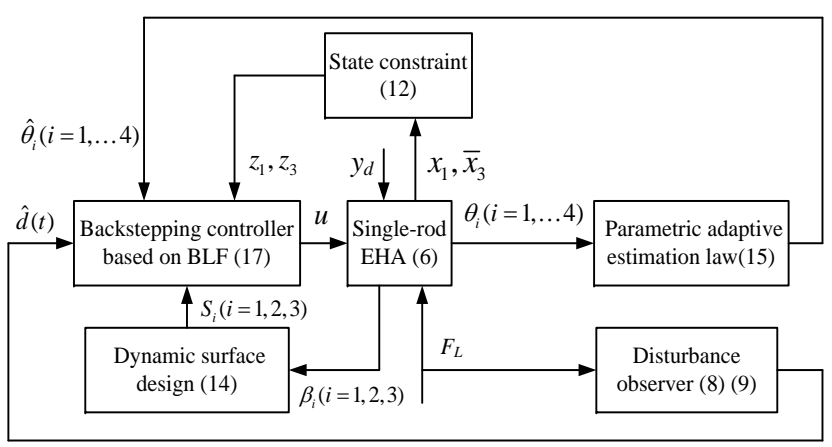

Fig. 3. Block diagram of the proposed control scheme

two double-acting cylinders (Hoerbiger LB6-1610-0080-4M), an axial piston pump (Takako TFH-315), a servo motor (BALDOR BSM63N-375), and a relief valve. The four cylinder pressures are measured by the pressure sensor (MEAS M3041000006-350BG). The two joint angles measured by the relative encoder (AVAGO AEDA-3300-BE1) are converted to the hydraulic positions of the upper arm and forearm respectively by triangular geometry.

The control algorithm is executed through the industrial personal computer (IPC). The load mass $m_{f}=1 \mathrm{~kg}$ is a disc on the top of the forearm. Some nominal hydraulic parameters of this EHS are shown in Tab. 1. The constrained boundaries of two state errors $z_{1}$ and $z_{3}$ are $k_{c 1}=10^{-3} \mathrm{~m}, k_{a 1}=-63$ bar, $k_{b 1}=37$ bar. The control gains are designed as $k_{1}=5000$, $k_{2}=500, k_{3}=10, k_{4}=1$. The observer gain is $K_{d}=100$. The parametric estimation gains are $k_{\theta 1}=10^{3}, k_{\theta 2}=3 \times 10^{9}$, $k_{\theta 3}=200, k_{\theta 4}=10^{6}$, The positive constants are $\sigma_{1}=10^{-3}$, $\sigma_{2}=10^{-2}, \sigma_{3}=10^{-2}, \sigma_{4}=5 \times 10^{-3}, \sigma_{5}=10^{9}, k=1000$. The time constants of the dynamic surfaces are $\tau_{1}=\tau_{2}=\tau_{3}$ $=10^{-3}$. The initial values of parametric estimations are $\hat{\theta}_{10}=$ $2000, \hat{\theta}_{20}=2.3 \times 10^{8}, \hat{\theta}_{30}=5.2 \times 10^{-3}, \hat{\theta}_{40}=1.5 \times 10^{5}$. The parametric uncertainty boundaries are $\theta_{1 \mathrm{~min}}=1500, \theta_{1 \max }=$ $3000, \theta_{2 \min }=2 \times 10^{8}, \theta_{2 \max }=3 \times 10^{8}, \theta_{3 \min }=5 \times 10^{-3}$, $\theta_{3 \max }=6 \times 10^{-3}, \theta_{4 \min }=1 \times 10^{5}, \theta_{4 \max }=2 \times 10^{5}$. Two sinusoidal demands of the cylinder displacements are $y_{1 d}=29 \sin (1.4 \pi t) \mathrm{mm}$ and $y_{2 d}=29 \sin (2 \pi t) \mathrm{mm}$.

TABLE I

THE HYDRAULIC PARAMETERS USED IN EXPERIMENTS

\begin{tabular}{llll}
\hline Parameter & \multicolumn{1}{c}{ Value } & Parameter & \multicolumn{1}{c}{ Value } \\
\hline$C_{d}$ & 0.62 & $w$ & $0.024 \mathrm{~m}$ \\
$x_{v \max }$ & $7.9 \mathrm{~mm}$ & $L_{\max }$ & $58 \mathrm{~mm}$ \\
$p_{s}$ & $40 \mathrm{bar}$ & $p_{r}$ & $2 \mathrm{bar}$ \\
$A_{a}$ & $2.01 \mathrm{~cm}^{2}$ & $A_{b}$ & $1.25 \mathrm{~cm}^{2}$ \\
$V_{t}$ & $3.417 \times 10^{-5} \mathrm{~m}^{3}$ & $\bar{\beta}_{e}$ & $2.2 \times 10^{8} \mathrm{~Pa}$ \\
$K_{s v}$ & $7.9 \times 10^{-4} \mathrm{~m} / \mathrm{V}$ & $T_{s v}$ & $12 \mathrm{~ms}$ \\
$K$ & $1000 \mathrm{~N} / \mathrm{m}$ & $\bar{b}$ & $2200 \mathrm{Ns} / \mathrm{m}$ \\
$\bar{C}_{t l}$ & $2.5 \times 10^{-11} \mathrm{~m}^{3} /(\mathrm{s} \cdot \mathrm{Pa})$ & $\bar{\rho}$ & $800 \mathrm{~kg} / \mathrm{m}^{3}$ \\
$m_{1 f}$ & $3.511 \mathrm{~kg}$ & $m_{2 f}$ & $1.739 \mathrm{~kg}$ \\
\hline
\end{tabular}

To illustrate the problem, the proposed controller is compared with the following two controllers.

1) PI controller $u=k_{p}\left(y_{d}-x_{1}\right)+k_{i} \int\left(y_{d}-x_{1}\right) d t$, where the control gains $k_{p}=100$ and $k_{i}=15$ have been well tuned to guarantee fast responses of two hydraulic actuators.

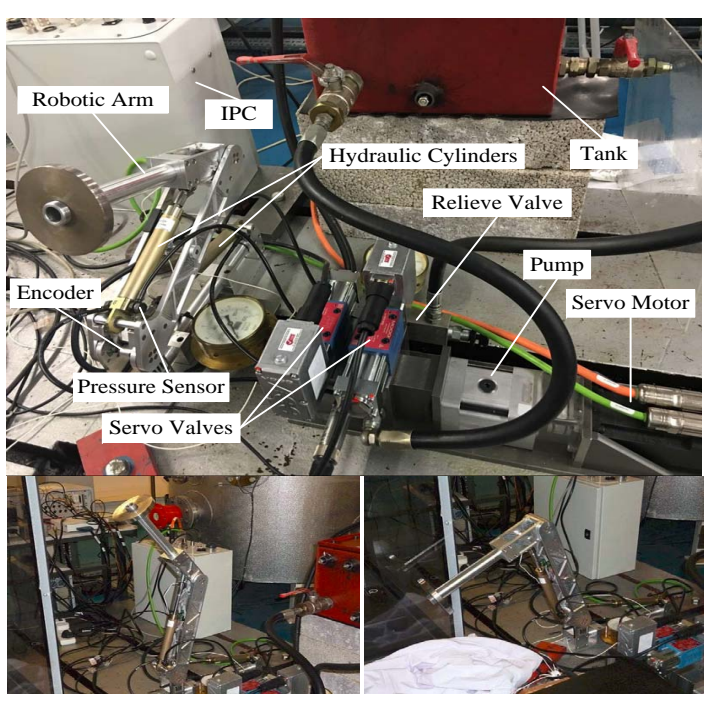

Fig. 4. The experimental bench and test scenario of the robotic arm

2) The general backstepping controller based on the Quadratic Lyapunov function and disturbance observer (QLFDOB) [8]

$$
\left\{\begin{aligned}
\hat{d} & =K_{d}\left(x_{2}-\xi\right) \\
\dot{\xi} & =f_{21}\left(x_{1}\right)+\bar{\theta}_{1} f_{22}\left(x_{2}\right)+g_{2} \bar{x}_{3}+\hat{d} \\
\alpha_{1} & =-k_{1} z_{1}+\dot{y}_{d} \\
\alpha_{2} & =\left(-k_{2} z_{2}-f_{21}-\bar{\theta}_{1} f_{22}-z_{1}+\hat{d}_{i}+\dot{\alpha}_{1}\right) / g_{2} \\
\alpha_{3} & =\left(-k_{3} z_{3}-g_{2} z_{2}-\bar{\theta}_{2} f_{31}-\bar{\theta}_{3} f_{32}+\dot{\alpha}_{2}\right) /\left(\bar{\theta}_{4} g_{3}\right) \\
u & =\left(-k_{4} z_{4}-\bar{\theta}_{4} g_{3} z_{3}-f_{4}+\dot{\alpha}_{3}\right) / g_{4}
\end{aligned}\right.
$$

which denotes that the virtual control derivatives $\dot{\alpha}_{i}(i=$ $1,2,3)$ are directly computed in the backstepping iteration.

Notably, this controller would not consider the stateconstrained problem and hydraulic parametric estimation. Thus, the nominal parameters $\bar{\theta}_{1}=\bar{b}, \bar{\theta}_{2}=\bar{\beta}_{e}, \bar{\theta}_{3}=\bar{\beta}_{e} \bar{C}_{t l}$, and $\bar{\theta}_{4}=\bar{\beta}_{e} C_{d} w \sqrt{2 / \bar{\rho}}$. In addition, the control gains of QLFDOB are the same as the proposed controller.

To evaluate the above three controllers, five performance indices are defined as the maximal position tracking error $e_{\max }=\max _{j=1, \ldots, n}\left\{\left|\Delta y_{i}(j)\right|\right\}$, the average position tracking error $\mu_{e}=\sum_{j=1}^{n} \Delta y_{i}(j) / n$, the standard deviation $\sigma_{e}=\sqrt{\sum_{j=1}^{n}\left(\Delta y_{i}(j)-\mu_{e}\right)^{2} /(n-1)}$, the boundaries of the load pressure $\bar{x}_{3 \min }=\max _{j=1, \ldots, n}\left\{\bar{x}_{3 i}(j)\right\}, \bar{x}_{3 \max }=$ $\min _{j=1 \ldots \ldots n}\left\{\bar{x}_{3 i}(j)\right\}$, where $n$ is the sample size of the above statistical variables.

The experimental results of three controllers are shown in Figs. 5-10 and the corresponding performance statistics are listed in Tab. 2. Fig. 5 shows that the dynamic tracking performance of the proposed controller $\left(e_{\max }=1.17 \mathrm{~mm}, \sigma_{e}\right.$ $=0.59 \mathrm{~mm})$ is better than the PI controller $\left(e_{\max }=3.69 \mathrm{~mm}\right.$, $\left.\sigma_{e}=1.49 \mathrm{~mm}\right)$ and the QLFDOB controller $\left(e_{\max }=2.89\right.$ $\left.\mathrm{mm}, \sigma_{e}=1.53 \mathrm{~mm}\right)$. However, certain discrepancies exist between the simulation results (see in supplemental material) 
and experimental results. For example, the experimental results $e_{\max }, \mu_{e}, \sigma_{e}$ of the proposed controller are obviously greater than those in the simulation. These discrepancies are caused by the measured error of the encoder. Since the encoder is 8 bit resolution, this means the actual measured accuracy of joint angle is $360^{\circ} / 2^{8}=1.4^{\circ}$. According to triangular geometry, the position tracking error caused by the measured error is approximately estimated by $\sqrt{a_{1}^{2}+b_{1}^{2}+2 a_{1} b_{1} \sin \left(1.4^{\circ}\right)}-$ $\sqrt{a_{1}^{2}+b_{1}^{2}}=1.1 \mathrm{~mm}$, where $a_{1}=0.322 \mathrm{~m}, b_{1}=0.045 \mathrm{~m}$. Thus, the experimental results of three controllers are often larger than the simulation due to measured errors which exist in different sensors.

There also exist partial chatters in the position response under the PI control, which indicates the tracking performance declines when the current external load disturbance on the upper arm approaches its limitation $\left(\hat{d}_{1}=150 \mathrm{~m} / \mathrm{s}^{2}\right)$ as shown in Fig. 9. Thus, the DO is designed to largely compensate dynamic load disturbance and to avoid the obvious chatter of the position response in the proposed controller and the QLFDOB controller. Furthermore, the proposed controller guarantees the faster load pressure response $\bar{x}_{3}$ and better tracking error $z_{1}$ than the QLFDOB controller.

From Fig. 6, the two load pressures periodically switch from positive to negative, which shows the extended and retracted motion of two cylinders. The positive maximum $\bar{x}_{3 \max }=$ 23.28 bar and the negative minimum $\bar{x}_{3 \min }^{\prime}=-37.44$ bar of load pressure by the proposed controller are obviously smaller than the PI controller $\left(\bar{x}_{3 \max }=41.92\right.$ bar, $\bar{x}_{3 \min }^{\prime}=-67.40$ bar) and the QLFDOB controller $\left(\bar{x}_{3 \max }=28.16\right.$ bar, $\bar{x}_{3 \min }^{\prime}=$ -45.28 bar). By the state-constrained control, the positive load pressures $\bar{x}_{3}$ on the two EHAs are in the boundary $p_{r}-v p_{s}<\bar{x}_{3}<p_{s}-v p_{r}$, i.e., $-22.87<\bar{x}_{3}<38.75$ bar, and the corresponding negative load pressures on the two EHAs $\bar{x}_{3}^{\prime}$ are in the boundary $p_{r}-p_{s} / v<\bar{x}_{3}^{\prime}<p_{s}-p_{r} / v$, i.e., $-62.32<\bar{x}_{3}^{\prime}<36.78$ bar.

The output errors of the dynamic surfaces $S_{i}(i=1,2,3)$ by the proposed controller are relatively smaller than the virtual control derivatives $\alpha_{i}(i=1,2,3)$ by the QLFDOB controller as shown in Figs. 7-8, which indicates that the stateconstrained controller (17) can avoid the violent control $u$ by $S_{i}$ instead of $\alpha_{i}$.

The load disturbance estimations on two EHAs by the proposed controller are shown in Fig. 9, which shows the external load on the upper arm is greater than that on the forearm, although the frequency of the former is smaller than the latter. The uncertainty parametric estimations are shown in Fig. 10. The four parametric estimations are regulated from the initial values to their nominal parameters respectively.

\section{CONCLUSION}

In this paper, a state-constrained control scheme was proposed for the single-rod EHA under hydraulic parametric uncertainty and external load disturbance. To restrict the position tracking error and the load pressure in the respective performance boundaries, this proposed controller is designed by BLF and integrated with dynamic surface, parametric and disturbance estimations. Furthermore, a disturbance observer
TABLE II

PERFORMANCE STATISTICS OF THREE CONTROLLERS IN EXPERIMENT

\begin{tabular}{cllll}
\hline Arm & Statistics & PI & QLFDOB & Proposed \\
\hline \multirow{5}{*}{ Upper arm } & $e_{\max }(\mathrm{mm})$ & 3.69 & 2.17 & 0.94 \\
& $\mu_{e}(\mathrm{~mm})$ & 0.06 & 0.14 & 0.16 \\
& $\sigma_{e}(\mathrm{~mm})$ & 1.21 & 1.29 & 0.47 \\
& $\bar{x}_{3 \max }(\mathrm{bar})$ & 41.92 & 28.16 & 23.28 \\
& $\bar{x}_{3 \min }^{\prime}(\mathrm{bar})$ & -67.40 & -45.28 & -37.44 \\
\hline \multirow{5}{*}{ Forearm } & $e_{\max }(\mathrm{mm})$ & 3.41 & 2.86 & 1.17 \\
& $\mu_{e}(\mathrm{~mm})$ & 0.02 & 0.11 & 0.16 \\
& $\sigma_{e}(\mathrm{~mm})$ & 1.49 & 1.53 & 0.59 \\
& $\bar{x}_{3 \max }(\mathrm{bar})$ & 27.37 & 20.62 & 18.10 \\
& $\bar{x}_{3 \min }^{\prime}(\mathrm{bar})$ & -44.02 & -33.16 & -29.10 \\
\hline
\end{tabular}
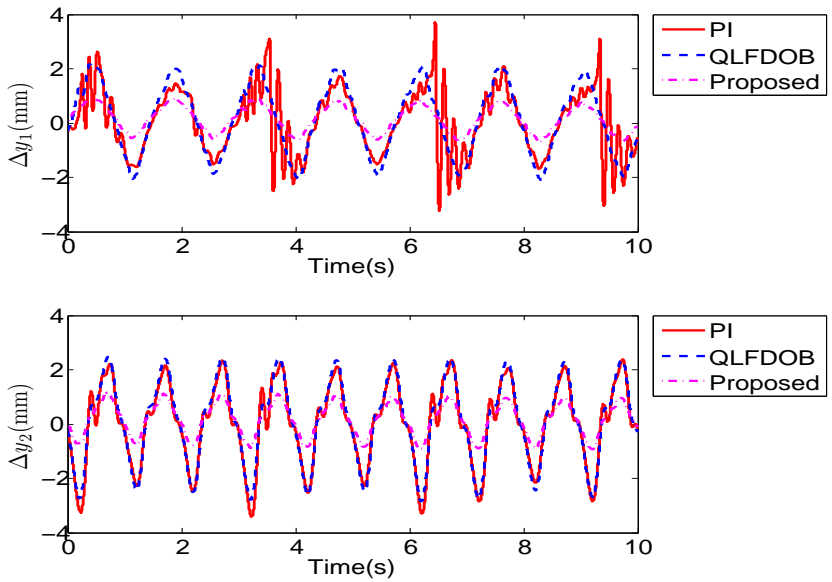

Fig. 5. The experimental results of position tracking errors by three controllers, $\Delta y_{1}-$ upper arm error, $\Delta y_{2}-$ forearm error
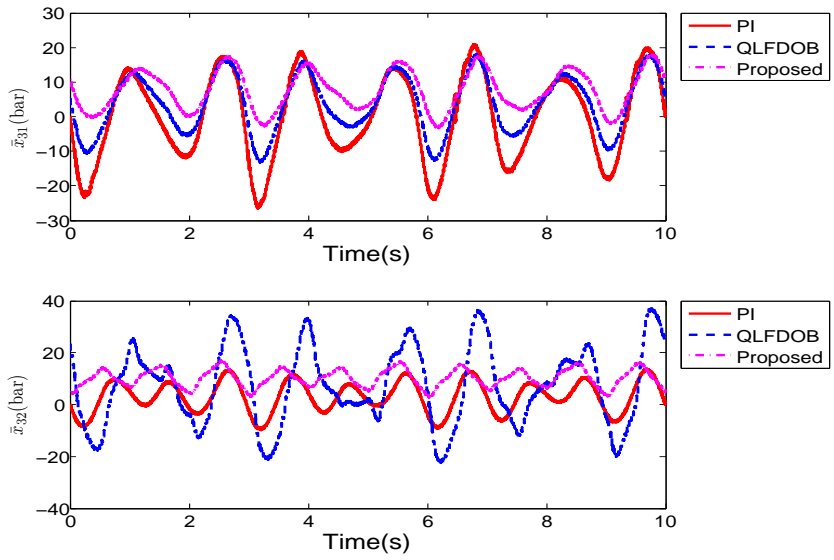

Fig. 6. The experimental results of hydraulic pressures by three controllers, $\bar{x}_{31}$ - load pressure on upper arm, $\bar{x}_{32}-$ load pressure on forearm

and a parametric estimation law are adopted to eliminate the zero bias of dynamic response and the negative effect of parametric uncertainty and the external load. Then three dynamic surfaces were used to avoid the explosion of complexity due to the repeatedly calculated differentiations of the virtual control. The comparison results with the other two controllers indicated that the the proposed controller can improve the dynamic performance of EHSs in practice. 

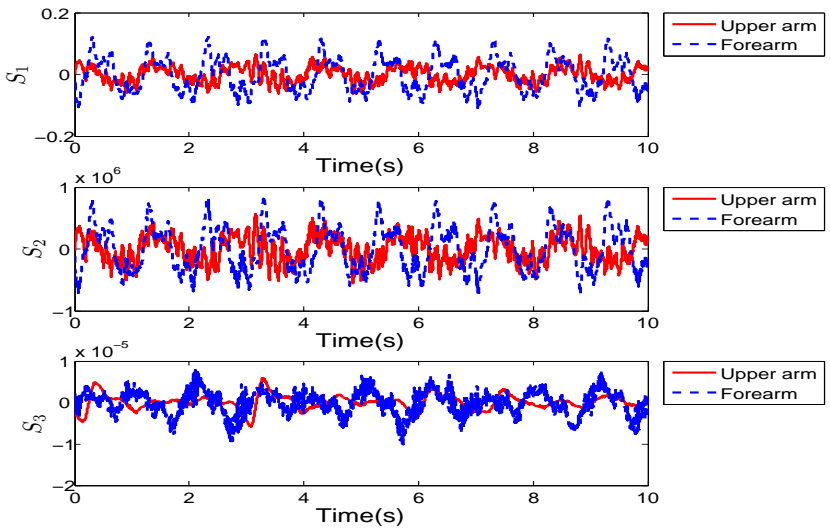

Fig. 7. The experimental results of the output errors of dynamic surfaces by the proposed controller
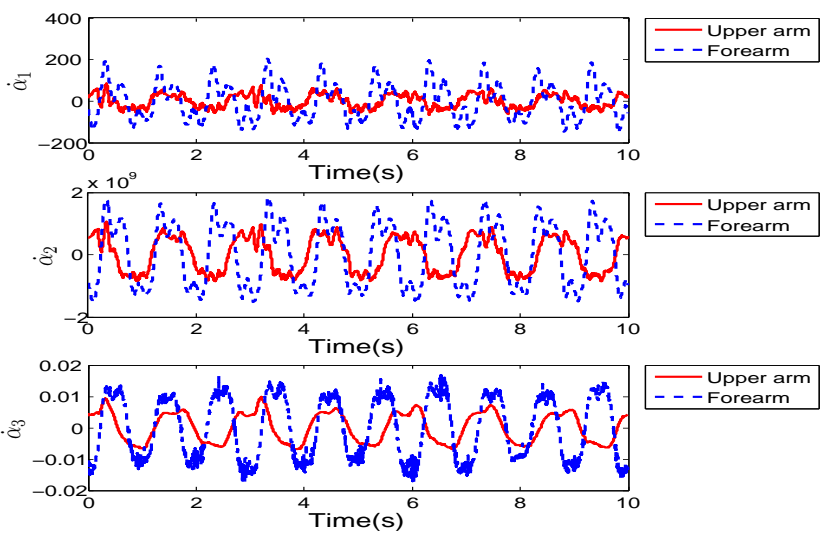

Fig. 8. The experimental results of the virtual control derivatives by the QLFDOB controller

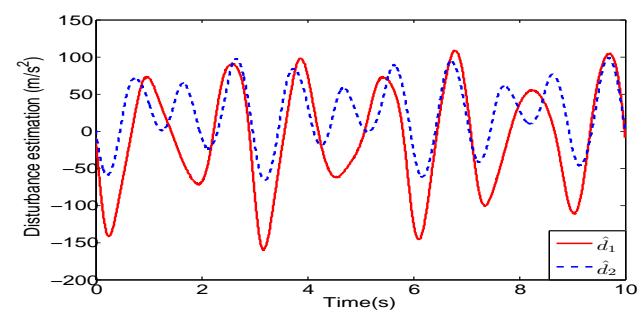

Fig. 9. The disturbance estimations on two hydraulic actuators by the proposed controller

\section{REFERENCES}

[1] H. Merritt, Hydraulic control systems. USA: John Wiley \& Sons, New York, NY, 1967

[2] B. Yao, F. Bu, J. Reedy, and G. T. C. Chiu, "Adaptive robust motion control of single-rod hydraulic actuators theory and experiments," IEEE/ASME Trans. Mechatronics, vol. 5, no. 1, pp. 79-91, Mar 2000.

[3] W. Chen, "Disturbance observer based control for nonlinear systems," IEEE/ASME Trans. Mechatronics, vol. 9, no. 4, pp. 706-710, Dec 2004.

[4] V. Milić, Željko Šitum, and M. Essert, "Robust $\mathrm{H}_{\infty}$ position control synthesis of an electro-hydraulic servo system," ISA Trans., vol. 49, no. 4, pp. 535-542, Oct 2010.

[5] X. Song, Y. Wang, and Z. Sun, "Robust stabilizer design for linear timevarying internal model based output regulation and its application to an electro hydraulic system," Automatica, vol. 50, no. 4, pp. 1128-1134, Apr 2014.

[6] C. Guan and S. Pan, "Nonlinear adaptive robust control of single-rod
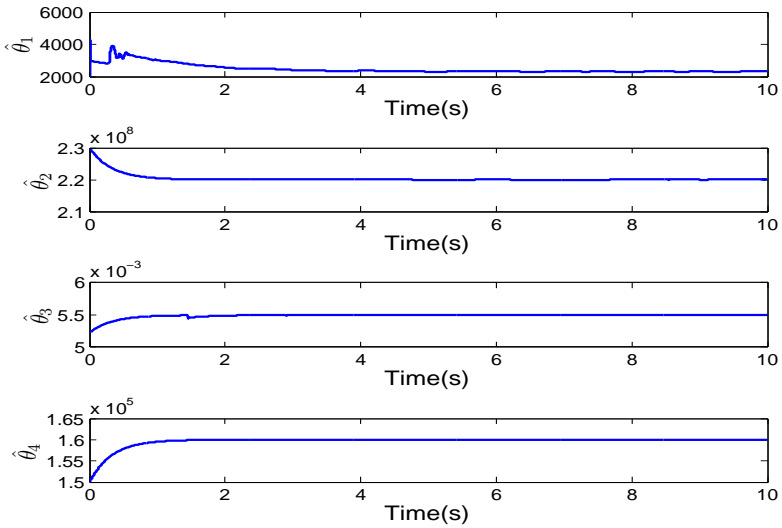

Fig. 10. The experimental results of uncertainty parametric estimations by the proposed controller

electro-hydraulic actuator with unknown nonlinear parameters," IEEE Trans. Control Syst. Technol., vol. 16, no. 3, pp. 434-445, May 2008.

[7] K. K. Ahn, N. C. N. Doan, and M. Jin, "Adaptive backstepping control of an electrohydraulic actuator," IEEE/ASME Trans. Mechatronics, vol. 19, no. 3, pp. 987-995, Jun 2014.

[8] Q. Guo, P. Sun, J. Yin, T. Yu, and D. Jiang, "Parametric adaptive estimation and backstepping control of electrohydraulic actuator with decayed memory filter," ISA Trans., vol. 62, pp. 202-214, Mar 2016.

[9] J. Yao, Z. Jiao, and D. Ma, "Extended-state-observer-based output feedback nonlinear robust control of hydraulic systems with backstepping," IEEE Trans. Ind. Electron., vol. 24, no. 6, pp. 993-1015, Apr 2014.

[10] K. P. Tee, S. S. Ge, and E. H. Tay, "Barrier lyapunov functions for the control of output-constrained nonlinear systems," Automatica, vol. 45, no. 1, pp. 918-927, Apr 2009.

[11] Y. Qiu, X. Liang, and Z. Dai, "Backstepping dynamic surface control for an anti-skid braking system," Control Eng. Pract., vol. 42, pp. 140-152, 2015.

[12] D. Won, W. Kim, D. Shin, and C. C. Chung, "High-gain disturbance observer-based backstepping control with output tracking error constraint for electro-hydraulic systems," IEEE Trans. Control Syst. Technol., vol. 23, no. 2, pp. 787-795, Mar 2015.

[13] L. Lu and B. Yao, "Online constrained optimization based adaptive robust control of a class of MIMO nonlinear systems with matched uncertainties and input/state constraints," Automatica, vol. 50, no. 3, pp. 864-873, Mar 2014.

[14] W. He, H. Huang, and S. S. Ge, "Adaptive neural network control of a robotic manipulator with time-varying output constraints," IEEE Trans. Cybern., vol. 47, no. 10, pp. 3136-3147, Oct 2017.

[15] W. He, A. O. David, Z. Yin, and C. Sun, "Neural network control of a robotic manipulator with input deadzone and output constraint," IEEE Trans. Syst., Man, Cybern. A, Syst., vol. 46, no. 6, pp. 759-770, Jun 2016.

[16] D. Wang and J. Huang, "Neural network-based adaptive dynamic surface control for a class of uncertain nonlinear systems in strict-feedback form,” IEEE Trans. Neural Netw., vol. 16, no. 1, pp. 195-202, Jan 2005.

[17] M. Hou and G. Duan, "Robust adaptive dynamic surface control of uncertain nonlinear systems,” Int. J. Control Autom. Syst., vol. 9, no. 1, pp. 161-168, Jan 2011.

[18] D. Swaroop, J. Hedrick, P. Yip, and J. Gerdes, "Dynamic surface control for a class of nonlinear systems," IEEE Trans. Automat. Contr., vol. 45, no. 10 , pp. $1893-1899$, Oct 2000.

[19] Moog, D633 and D634 Series Direct Drive Servo-Proportional Control Valves. [Online]. Available: http://www.moog.com.cn/literature/ICD/ Moog-Valves-D633_D634-Catalog-chs.pdf

[20] B. Ren, S. S. Ge, K. P. Tee, and T. H. Lee, "Adaptive neural control for output feedback nonlinear systems using a barrier lyapunov function," IEEE Trans. Neural Netw., vol. 21, no. 8, pp. 1339-1345, Aug 2010.

[21] E. Sontag, Mathematical control theory. In Texts in Applied Mathematics: Deterministic finite-dimensional systems, 2nd ed. USA: Springer-Verlag, New York, NY, 1998.

[22] W. Sun, H. Gao, and O. Kaynak, "Vibration isolation for active suspensions with performance constraints and actuator saturation," IEEE/ASME Trans. Mechatronics, vol. 20, no. 2, pp. 675-683, Apr 2015. 